\title{
The effectiveness of acupuncture on the sleep quality of elderly with dementia: a within-subjects trial
}

This article was published in the following Dove Press journal:

Clinical Interventions in Aging

30 July 2013

Number of times this article has been viewed

\author{
Timothy Kwok ${ }^{1,2}$ \\ Ping Chung Leung ${ }^{3}$ \\ Yun Kwok Wing ${ }^{4}$ \\ Isaac Ip ${ }^{2}$ \\ Bel Wong ${ }^{2}$ \\ Daniel Wai Hung $\mathrm{Ho}^{2}$ \\ Wai Ming Wong ${ }^{3}$ \\ Florence $\mathrm{Ho}^{2}$ \\ 'Department of Medicine and \\ Therapeutics, The Chinese University \\ of Hong Kong, Hong Kong; ${ }^{2}$ Jockey \\ Club Centre for Positive Ageing, \\ Hong Kong; ${ }^{3}$ Institute of Chinese \\ Medicine, ${ }^{4}$ Department of Psychiatry, \\ The Chinese University of Hong Kong, \\ Hong Kong
}

Correspondence: Bel Wong Jockey Club Centre for Positive Ageing, 27 A Kung Kok Street, Shatin,

NT, Hong Kong

Tel +852 26366323

Fax +852 26360323

Email research@jccpahk.com
Purpose: Elderly with dementia are often afflicted with sleep problems. Recent studies have suggested that acupuncture may be a feasible alternative to traditional sleep medicine for treating sleep disturbance. This study investigated the effectiveness of acupuncture on sleep quality of elderly with dementia.

Patients and methods: Nineteen elders with dementia were followed through a control period and an acupuncture treatment period, each lasting 6 weeks. Outcome measures were subjects' sleep quality and cognitive function. Sleep parameters were recorded by wrist actigraphy. Cognitive function was assessed by the Alzheimer's Disease Assessment Scale - Cognitive (ADAS-Cog). Pretests and posttests were conducted immediately before and after the control and treatment periods. Changes in the outcome measures between control and treatment periods were compared.

Results: Wilcoxon signed rank tests revealed that the subjects gained significantly more resting time and total sleep time in the treatment period than in the control period $(P<0.05)$. A nonsignificant trend for improvement in sleep efficiency was observed. Improvement in cognitive function was not statistically significant. A total of $86 \%$ of the subjects completed the treatment regime.

Conclusion: Results reveal that acupuncture was effective in improving some domains of sleep quality of elderly with dementia, and the subjects showed acceptance towards the intervention. Strengths and limitations of the present study as well as suggestions for further studies were considered.

Keywords: acupuncture, sleep disturbance, patients with dementia

\section{Introduction}

Elderly with dementia are often afflicted with sleep disturbances. Cross-sectional studies of community-residing samples revealed that $19 \%-50 \%$ of elderly who developed dementia experienced disruptions in their sleep. ${ }^{1-3}$ Moreover, sleep disruption appears to worsen as the disease progresses; ${ }^{4}$ it has been estimated that elderly in the later stages of dementia spend as much as $40 \%$ of their time in bed awake. ${ }^{5}$ Sleep disturbance is generally defined as having trouble falling asleep and maintaining sleep at any given time. Particularly, the sleep pattern of dementia patients is often marked by increased wakefulness at night (increases in time awake and number of awakenings); reduced slow-wave sleep (SWS) and rapid eye movement (REM) sleep; and increased daytime sleepiness. $^{6-8}$

Research has shown that poor sleep quality can diminish the well-being of seniors with dementia, particularly in aspects of cognitive impairment, incidence of physical 
illness, and development of psychological disorders. ${ }^{5}$ Moreover, the high prevalence of sleep problems can be a significant source of physical and psychological distress to caregivers, ${ }^{6}$ and thus causes negative impact on the quality of life of both caregivers and care recipients. From the public health perspective, the common occurrence of sleep disturbance has become a pressing concern among the dementia population.

In response to the negative consequences of poor sleep, studies $^{7,11-13,15}$ have investigated the effectiveness of various interventions to enhance sleep quality of people with dementia. Traditional sedating medications such as benzodiazepine and antihistamines are common prescriptions for dementia patients with sleep problems. Some of these medications, however, can cause detrimental side effects, including excessive daytime sleepiness and impaired motor performance. ${ }^{7}$ In addition, pharmacological treatments also give rise to the issue of polypharmacy in elderly patients with dementia, which has been shown to be associated with functional decline. ${ }^{8}$ In this respect, non-pharmacological interventions have emerged as a viable alternative for treating sleep disturbance in this group of patients.

Acupuncture is an effective non-pharmacological therapeutic technique in treating sleep problems. It is a form of traditional Chinese medicine (TCM) that essentially corrects health problems by the use of sterilized needles inserted into meridian points of the human body. According to TCM theory, pain or diseases are a result of an imbalance in the yin and yang forces in the body or of excess or deficiency of Qi (air), blood, or body fluids. The mechanisms of acupuncture work by recreating the balance in the yin and yang forces and restoring Qi, blood, and body fluids to normalcy through stimulation of specific meridian points that govern different organs and their interactions. ${ }^{9}$ The procedure is considered safe, provided that it is handled by well-trained therapists and that necessary safety precautions are followed. ${ }^{10}$ As summarized from various literature, the common meridian points for sleep promotion are Shenmen (HT7), Baihui (DU20), Anmian II (EX-HN9), Yintang (EX-HN3), Zusanli (ST36), and Sanyinjiao (SP6); the treatment duration generally spans from 3 to 6 weeks. ${ }^{11-15}$

Empirical evidence has shown that acupuncture produces positive outcomes in alleviating sleep disturbances in patients with HIV disease and renal diseases. ${ }^{11,13}$ The therapy is also used as intervention in the elderly for diverse aging conditions, such as agitated behavior and cognitive impairment. One study showed that the use of acupuncture could reduce agitated behaviors exhibited by dementia patients, thus resulting in better sleep quality. ${ }^{14}$ Another trial demonstrated that a 5-week acupuncture intervention improved the sleep quality of anxious adult subjects by causing an increase in melatonin secretion, ${ }^{16}$ a hormone that plays a role in regulating the sleep-wake cycle. Studies suggest that melatonin might help restore the circadian modulation of REM sleep in people with REM sleep behavior disorder, and thereby normalize REM sleep. ${ }^{17,18}$ It has been shown that melatonin improved the REM sleep quality of patients with schizophrenia, while hormone levels can be increased by acupuncture treatment. ${ }^{19}$ Deficiency of melatonin is associated with insomnia in elderly people. ${ }^{20}$ The positive evidence for the therapeutic value of acupuncture warranted further investigation.

The present research aimed to develop an acupuncture program for enhancing sleep quality in seniors with dementia and to evaluate its effectiveness in reducing sleep disturbances by adopting a within-subjects design. It was expected that subjects would exhibit less sleep disturbances and show improvements in sleep quality after the acupuncture intervention.

\section{Methods}

\section{Subjects}

Twenty-two elders (aged between 55-90 years old) diagnosed with dementia who had sleep disturbances were recruited by convenience sampling from elderly community centers, dementia-related health talks, and the memory clinic of a dementia service center. Inclusion criteria included (1) clinical diagnosis of dementia, (2) subjective complaints of sleep problems, and (3) stable drug regime or no new drug intake in the 3 months prior to the study. Exclusion criteria were skin problems around the application area, psychiatric disorder, or aggressive behavior. Informed consent to participate was obtained from family caregivers. The treatment procedure was explained orally to the subjects. Three subjects were unable to complete the treatment due to illness unrelated to their sleep problems; 19 subjects completed the treatment (completion rate being 86\%).

\section{Procedure}

The study took place between March 2011 and August 2012 using a within-subjects design. Subjects were assigned to batches according to the dates they enrolled in the study, so that each batch consisted of three to five elders. Each batch of subjects went through a 6-week wait-list control period and then a 6-week treatment period, one batch followed by another. Assessments on sleep quality and cognitive functioning were 
carried out immediately before and after control and treatment periods. During the treatment period, one-on-one acupuncture treatment was offered to the subjects. The treatment took place at a dementia service center and was carried out by a registered Chinese medicine practitioner with considerable clinical experience in acupuncture. Subjects were accompanied by their caregivers during the acupuncture sessions. Frequency of treatment was twice a week for 6 weeks (a total of 12 sessions was offered to each subject), similar to that in a previous trial. ${ }^{16}$ Each treatment session lasted approximately 30 minutes. Treatment was applied on six meridian points, namely, Shenmen (HT7), Baihui (DU20), Anmian II (EXHN9), Yintang (EX-HN3), Zusanli (ST36), and Sanyinjiao (SP6). For better experimental control, acupuncture was only applied to these six meridian points for each subject throughout all treatment sessions.

\section{Measures}

Sleep disturbance was measured by part K (sleep and nighttime behavior disorders) of the Chinese version of the Neuropsychiatric Inventory (NPI). ${ }^{21,22}$ Caregivers reported the presence of nighttime behaviors of the elders on eight items (eg, getting up during the night, dressing and undressing repeatedly) and rated the overall sleep problem in terms of frequency (from 1 = occasionally or less than once a week to 4 = very frequently, once or more per day, or continuously) and severity ( $1=$ mild, $2=$ moderate, $3=$ severe $)$. A domain score was formed by multiplying the frequency and severity scores. Higher scores represented more serious sleep disturbance.

Sleep parameters were measured by wrist actigraphy (Actiwatch 2, Respironics, Inc., Murrysville, PA, USA), which was a sufficiently sensitive method by which to detect the effects of intervention targeting insomnia in elderly subjects. ${ }^{23,24}$ The device detected the wrist movement of the wearer and recorded data for a number of sleep parameters. "Resting time" was the period of time (in minutes) between the subject going to bed at night and rising from bed in the morning. This information was recorded by the caregivers by pressing a button on the Actiwatch at the beginning and end of the period. "Total sleep time" was the total time (in minutes) of "stage of falling asleep" (movement of the subject as detected by Actiwatch) of individual subject within the "Resting time". "Onset latency" was the period of time (in minutes) between the start of resting time and sleep time. "Sleep efficiency" was the percentage of total sleep time over resting time. "Wake after sleep onset" (WASO) was the amount of waking minutes within total sleep time.
"Awake number" was the number of continuous blocks of wake within total sleep time. Sleep statistics were recorded for three consecutive nights at each assessment time point for more reliable measurement. ${ }^{25}$ Measures of each sleep parameter were yielded by taking the average of each sleep statistic across three nights.

Cognitive function was measured by the Chinese version of the Alzheimer's Disease Assessment Scale - Cognitive (ADAS-Cog). ${ }^{25,26}$ The ADAS-Cog is a widely adopted penand-paper test designed for the evaluation of cognitive deficits in persons with dementia. Subscales of the ADAS-Cog examined were list-learning, delayed recall, naming, ideational apraxia, construction, and recognition. The test was administered by trained research assistants, and had a maximum possible score of 70 and minimum of 0 , with increasing scores indicating more severe cognitive impairment.

\section{Data analysis}

Statistical analyses were carried out using SPSS software (version 20; IBM Corporation, Armonk, NY, USA). Data were analyzed on two levels. On the first level, differences between pretests and posttests in treatment and control periods were compared using the Wilcoxon signed-rank test. On the second, results from the treatment period were compared against the control period. Thus, changes between pretest and posttest scores were first calculated then compared between the treatment and control periods using the Wilcoxon signedrank test.

\section{Results \\ Demographics and baseline characteristics of subjects}

The majority of subjects were aged between 75 to 84 years (63.2\%) at baseline. Most subjects were female (73.7\%); more than half were widowed (57.9\%) and $42.1 \%$ were married. Most subjects had graduated from primary school (36.8\%) and secondary school or above (31.6\%). All but one subject $(94.7 \%)$ was cared for by family members or a domestic helper. According to the classification of Diagnostic and Statistical Manual of Mental Disorders, Fourth Edition, Text Revision (DSM-IV-TR), ${ }^{27}$ five of the subjects (26.3\%) had dementia of the Alzheimer's type; five (26.3\%), dementia due to other general medical conditions; three (15.8\%), dementia due to multiple etiologies; one (5.3\%), vascular dementia; and one (5.3\%), dementia due to Parkinson's disease (5.3\%), while four subjects did not provide their clinical diagnosis on dementia. Four subjects (21.1\%) were under a stable prescription of drugs related to sleep regulation. 
At baseline, the subjects had a mean resting time of 521.04 minutes (standard deviation [SD] 81.23) and sleeping time of 390.73 minutes (SD 121.21) at night; average sleep efficiency was 69.9\% (SD 18.4). Mean ADAS-Cog score was 27.28 (SD 10.93), and mean NPI-part K domain score was 3.92 (SD 3.65). Frequency of sleep disturbances and nighttime behaviors reported by caregivers are summarized in Table 1.

\section{Acceptability to the intervention}

Since this study adopted convenience sampling, no refusal was encountered. After recruitment, there were 19 of $22(86 \%$ success rate) subjects who completed the intervention. The reasons for withdrawal were intercurrent illness, perceived lack of response, and fear of needles.

Throughout the intervention, all but one subject attended and completed all sessions on schedule. One subject did not attend the last session due to illness not related to the intervention.

\section{Sleep quality of subjects}

Wilcoxon signed-rank test showed that, during the treatment period, increase in total sleep time and resting time was statistically significant $(Z=-2.13, P<0.05$ and $Z=-1.97$, $P<0.05$, respectively). Changes in other sleep and cognitive parameters (sleep efficiency, onset latency, WASO, awake number, and ADAS-Cog) during the treatment period were not statistically significant. As for control period, no significant difference was observed between pretests and posttests in any of the sleep and cognitive parameters.

Results of changes of sleep parameters and cognitive ability of the treatment period against the control period

Table I Sleep disturbances and nighttime behaviors on the NPI reported by caregivers

\begin{tabular}{ll}
\hline & $\%$ \\
\hline Disturbances & 76.2 \\
Difficulty falling asleep & 71.4 \\
Getting up during the night & 61.9 \\
Awaking too early in the morning & 28.6 \\
Other nighttime behaviors & 23.8 \\
Wandering, pacing, or inappropriate activities at night & 19.0 \\
Waking at night, dressing, and planning to go out & 14.3 \\
Dressing and undressing repeatedly & 78.9 \\
Response statistics & Number \\
Two or more of the above disturbances & 3.1 \\
\hline Mean number of sleep disturbances & 3.5 \\
\hline Median number of sleep disturbances &
\end{tabular}

Abbreviation: NPI, Neuropsychiatric Inventory. indicated that gain in resting time of the subjects during treatment period ( $\triangle M=42.05, \mathrm{SD}=86.61)$ was significantly greater than that during the control period $(\triangle M=-45.21$, $\mathrm{SD}=98.10 ; Z=-2.05, P<0.05$; see Figure 1). Similarly, gain in total sleep time of the subjects during the treatment period $(\triangle M=42.02, \mathrm{SD}=80.85)$ was significantly greater than during the control period $(\triangle M=-32.95, \mathrm{SD}=96.61$; $Z=-2.21, P<0.05$; see Figure 2). Gain in sleep efficiency during the treatment period $(\triangle M=2.60, \mathrm{SD}=13.58)$ was also greater than that during the control period $(\triangle M=-2.27$, $\mathrm{SD}=10.89$ ), although the difference was nonsignificant $(Z=-1.57, P=0.12)$. No significant differences were observed in changes in ADAS-Cog score and other sleep parameters (onset latency, WASO, and awake number) between the treatment and control periods (Table 2).

\section{Discussion}

Using a within-subjects design, the present study investigated the effectiveness of acupuncture on treating sleep disturbance of elderly with dementia. Results show that the intervention elicited significant improvement in sleep quality of elders with dementia in terms of significant gain in resting time as well as sleep time in the treatment period over the control period. A trend in improvement of sleep efficiency was also evident, albeit nonsignificant.

The acupuncture treatment resulted in significant improvement in total sleep time (time in bed that subjects actually spent sleeping). This result is consistent with the finding of a previous study that acupuncture treatment led to increased release of melatonin, ${ }^{16}$ which functioned to regulate the sleep-wake cycle. Interestingly, increase in sleep time in our subjects did not result in significantly better sleep efficiency. As sleep efficiency was calculated as the percentage of sleep time over resting time, the absence of significant findings could be due to the fact that resting time was also lengthened, hence "balanced out" the increase in sleep time.

The findings of increased resting time after acupuncture might be interpreted in various ways. Two meridian points (Baihui, DU20; Yintang, EX-HN3) were included in the intervention protocol specifically for relaxation and calmness. In general, subjects felt more relaxed during the intervention; this might have resulted in an increase in resting time. In addition, since the caregivers were not blinded to the acupuncture treatment, the caregivers might have adjusted the daily life of the subjects, eg, by arranging a longer resting time for the sake of the subjects. While it is implausible to blind the caregivers or subjects to acupuncture treatment, future trials 


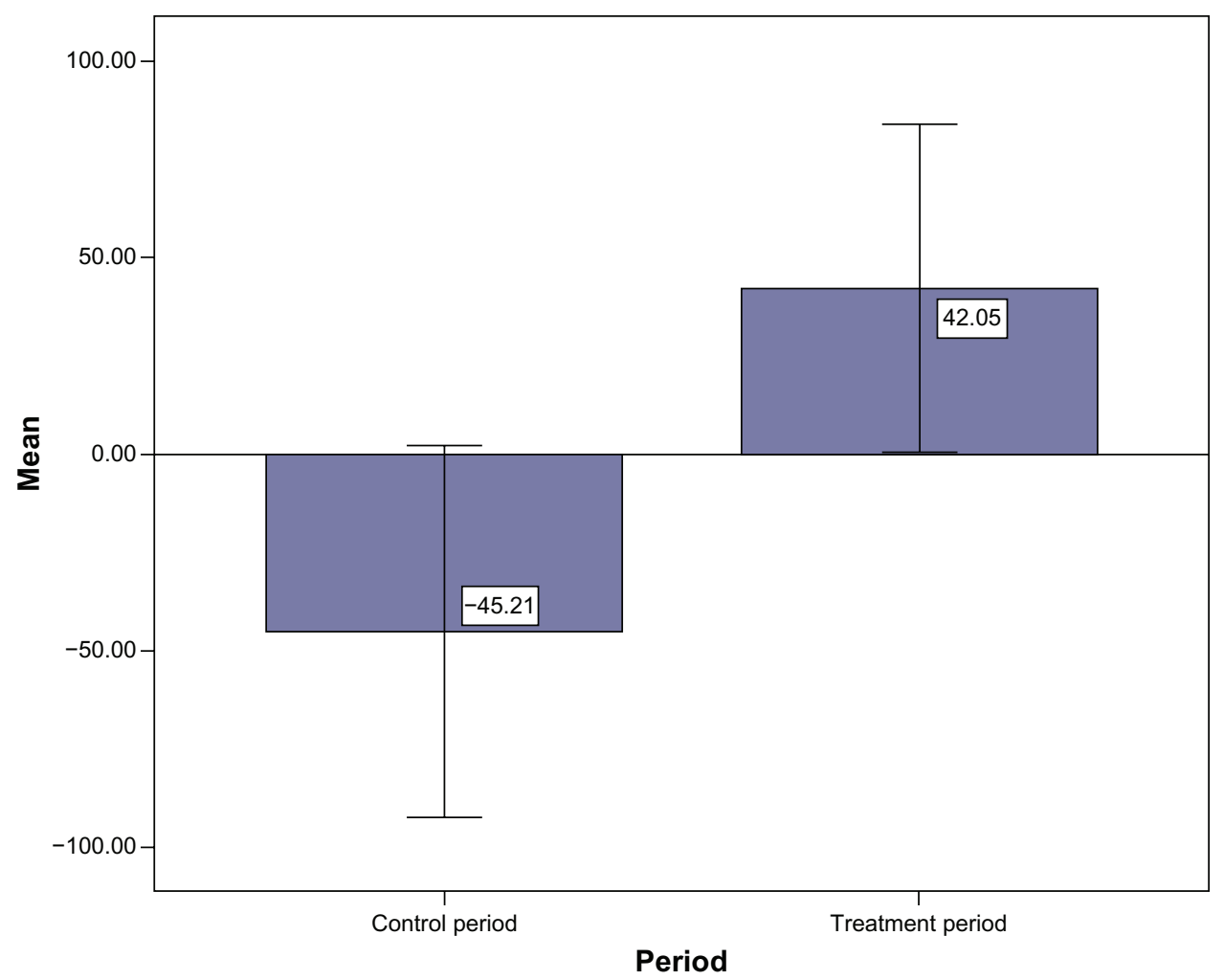

Figure I Changes in resting time (time when the subject went to bed with the intent to sleep) during the control period ( $\triangle M=-45.2 \mathrm{I})$ and treatment period $(\triangle M=42.05)$.

Note: Times are in minutes.

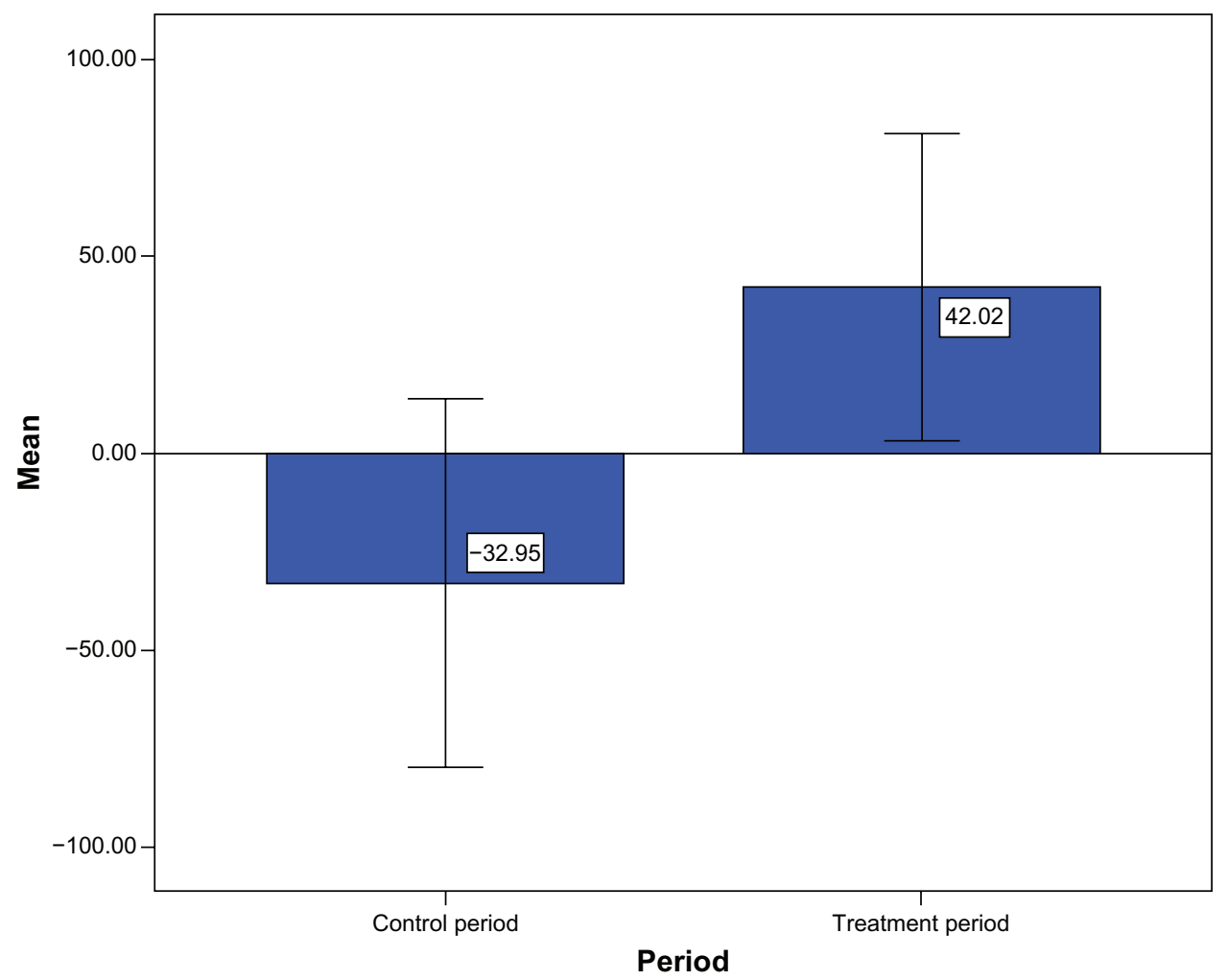

Figure 2 Changes in total sleep time (total time within rest intervals actually spent sleeping) during control period $(\triangle M=-32.95)$ and treatment period $(\triangle M=42.02)$. Note: Times are in minutes. 
Table 2 Comparison of mean differences in pre- and posttreatment/control periods of sleep and cognitive parameters

\begin{tabular}{|c|c|c|c|c|c|}
\hline \multirow[t]{2}{*}{ Outcome measure } & \multicolumn{2}{|c|}{$\begin{array}{l}\text { Treatment } \\
\text { period }\end{array}$} & \multicolumn{2}{|c|}{$\begin{array}{l}\text { Control } \\
\text { period }\end{array}$} & \multirow{2}{*}{$\begin{array}{l}\text { Wilcoxor } \\
Z\end{array}$} \\
\hline & $\triangle M$ & SD & $\triangle M$ & SD & \\
\hline Resting time & 42.05 & 86.61 & -45.21 & 98.1 & $-2.05^{*}$ \\
\hline Total sleep time & 42.02 & 80.85 & -32.95 & 96.61 & $-2.21^{*}$ \\
\hline Sleep onset latency & 3.76 & 34.51 & 1.02 & 21.49 & -0.04 \\
\hline Sleep efficiency & 2.60 & 13.58 & -2.27 & 10.89 & -1.57 \\
\hline Wake after sleep onset & -1.04 & 73.79 & -12.28 & 62.33 & -0.24 \\
\hline Awake number & -1.96 & 21.31 & -6.93 & 1.96 & -0.64 \\
\hline ADAS-Cog & -0.56 & 4.13 & 1.73 & 7.81 & -0.89 \\
\hline
\end{tabular}

Note: $* P<0.05$; times are in minutes.

Abbreviations: ADAS-Cog, Alzheimer's Disease Assessment Scale - Cognitive; $S D$, standard deviation.

might include a sham acupuncture group or sham acupuncture period to rule out this confounding factor.

Measures such as onset latency, WASO, and awake number did not show significant changes compared to control period. This could be due to the relatively less intense treatment schedule compared to previous studies. For instance, a trial conducted on institutionalized residents in Taiwan provided acupressure treatment on five consecutive days per week for 3 weeks and found significant improvements in latency and sleep efficiency, in addition to sleep time. ${ }^{28}$ In the present study, almost all the subjects (95\%) were community residing; to implement such an intensive treatment schedule would be impractical and create a heavy burden on the caregivers, as they needed to accompany the elders in travel to and from the service center. Future studies are needed to further investigate the optimal duration and intensity of an acupuncture treatment schedule.

The figures related to recruitment and intervention progress reflect that the subjects accepted the intervention and could manage to tolerate it. Only one subject in the study dropped out of the intervention because of fear of acupuncture.

It is generally believed that acupuncture is not welcome due to fear of needles. A study on acceptability of acupuncture on low back pain concluded that discomfort towards the intervention could be mediated by a good therapeutic relationship and clear explanations of the anticipated treatment outcome. ${ }^{29}$ The American Medical Association also suggests that information such as treatment options, expected outcomes, and relative risks should be given to the patients before treatment in a culturally and linguistically appropriate manner. ${ }^{30}$

\section{Strengths and limitations}

A recent Cochrane Review highlighted the small number of rigorous efficacy studies currently available in the literature on the effectiveness of acupuncture in treating sleep disturbance. ${ }^{10}$ Due to various limitations, few trials with rigorous research design on dementia patients have been conducted. For instance, a previous trial that found melatonin increase following acupuncture treatment did not include a control group. ${ }^{20}$ By using a within-subjects design with control and treatment conditions, the subjects in this study served as their own control. We were therefore able to determine the effects of acupuncture on sleep quality while reducing undesirable variance due to individual differences, which might be a potential shortcoming in other between-subjects trials with a relatively small sample size.

The present study was, nonetheless, subjected to several limitations. First, there was no significant difference observed on the outcome measures across demographic characteristics such as dementia type or drug intake; given the small sample size, the present study might lack sufficient statistical power to detect significant findings. Generalizability of the present findings was also limited. Second, although this study compared subjects' sleep quality during the control period versus the treatment period, it did not introduce a sham acupuncture period or contain a sham control group, hence the possibility of placebo effect could not be ruled out. Third, there lacked posttreatment follow-up assessment, thus the long-term effect of the acupuncture intervention was not investigated in the present study.

\section{Conclusion}

Using a within-subjects design, this pilot study provided preliminary but promising evidence for the favorable effect of acupuncture on the sleep quality of elderly with dementia. Acupuncture treatment led to significant improvements in terms of resting time and sleep time. Taking into consideration the strengths and limitations of this pilot study, acupuncture is suggested to be a viable alternative to traditional sleep mediation for treating sleep disturbances in this group of patients, while further studies are suggested to investigate the effects of various intensities in treatment schedule and the lasting effect of acupuncture intervention with a larger sample size and by including follow-up assessments. A proper sham acupuncture control group or sham acupuncture period (if not a randomized, controlled trial) should be included in research design to rule out potential placebo effect.

\section{Disclosure}

The authors report no conflicts of interest in this work. 


\section{References}

1. Hart DJ, Craig D, Compton SA, et al. A retrospective study of the behavioural and psychological symptoms of mid and late phase Alzheimer's disease. Int J Geriatr Psychiatry. 2003;18(11): 1037-1042.

2. McCurry SM, Gibbons LE, Logsdon RG, Vitiello MV, Teri L. Nighttime insomnia treatment and education for Alzheimer's disease: a randomized, controlled trial. J Am Geriatr Soc. 2005;53(5):793-802.

3. Shub D, Darvishi R, Kunik ME. Non-pharmacologic treatment of insomnia in persons with dementia. Geriatrics. 2009;64(2):22-26.

4. Pat-Horenczyk R, Klauber MR, Shochat T, Ancoli-Israel S. Hourly profiles of sleep and wakefulness in severely versus mild-moderately demented nursing home patients. Aging (Milano). 1998;10(4): 308-315.

5. Buysse DJ, Reynolds CF 3rd, Monk TH, Berman SR, Kupfer DJ. The Pittsburgh Sleep Quality Index: a new instrument for psychiatric practice and research. Psychiatry Res. 1989;28(2):193-213.

6. Pinquart M, Sörensen S. Differences between caregivers and noncaregivers in psychological health and physical health: a metaanalysis. Psychol Aging. 2003;18(2):250-267.

7. Richardson GS, Roehrs TA, Rosenthal L, Koshorek G, Roth T. Tolerance to daytime sedative effects of $\mathrm{H} 1$ antihistamines. J Clin Psychopharmacol. 2002;22(5):511-515.

8. Lau DT, Mercaldo ND, Shega JW, Rademaker A, Weintraub S. Functional decline associated with polypharmacy and potentially inappropriate medications in community-dwelling older adults with dementia. Am J Alzheimers Dis Other Demen. 2011;26(8):606-615.

9. Maciocia G. Foundations of Chinese Medicine: A Comprehensive Text for Acupuncturists and Herbalists. Edinburgh: Churchill Livingston; 1989.

10. Cheuk DK, Yeung WF, Chung KF, Wong V. Acupuncture for insomnia. Cochrane Database Syst Rev. 2007;(3):CD005472.

11. Phillips KD, Skelton WD. Effects of individualized acupuncture on sleep quality in HIV disease. J Assoc Nurses AIDS Care. 2001;12(1): 27-39.

12. Suen LKP, Wong TKS, Leung AWN. Effectiveness of auricular therapy on sleep promotion in the elderly. Am J Chin Med. 2002;30(04): 429-449.

13. Tsay SL, Chen ML. Acupressure and quality of sleep in patients with end-stage renal disease: a randomized controlled trial. Int J Nurs Stud. 2003;40(1):1-7.

14. Yang MH, Wu SC, Lin JG, Lin LC. The efficacy of acupressure for decreasing agitated behaviour in dementia: a pilot study. J Clin Nurs. 2007;16(2):308-315.

15. Sjöling M, Rolleri M, Englund E. Auricular acupuncture versus sham acupuncture in the treatment of women who have insomnia. J Altern Complement Med. 2008;14(1):39-46.
16. Spence DW, Kayumov L, Chen A, et al. Acupuncture increases nocturnal melatonin secretion and reduces insomnia and anxiety: a preliminary report. J Neuropsychiatry Clin Neurosci. 2004;16(1):19-28.

17. Cajochen C, Kräuchi K, Möri D, Graw P, Wirz-Justice A. Melatonin and S-20098 increase REM sleep and wake-up propensity without modifying NREM sleep homeostasis. Am J Physiol. 1997;272(4 Pt 2): R1189-R1196.

18. Kunz D, Bes F. Melatonin as a therapy in REM sleep behavior disorder patients: an open-labeled pilot study on the possible influence of melatonin on REM-sleep regulation. Mov Disord. 1999;14(3): 507-511.

19. Bosch P, van den Noort M, editors. Schizophrenia, Sleep, And Acupuncture. Germany: Hogrefe Publishing GmbH; 2008.

20. Garfinkel D, Laudon M, Nof D, Zisapel N. Improvement of sleep quality in elderly people by controlled-release melatonin. Lancet. 1995;346(8974):541-544.

21. Cummings JL, Mega M, Gray K, Rosenberg-Thompson S, Carusi DA, Gornbein J. The Neuropsychiatric Inventory: comprehensive assessment of psychopathology in dementia. Neurology. 1994;44(12):2308.

22. Leung VP, Lam LC, Chiu HF, Cummings JL, Chen QL. Validation study of the Chinese version of the neuropsychiatric inventory (CNPI). Int J Geriatr Psychiatry. 2001;16(8):789-793.

23. Ancoli-Israel S, Clopton P, Klauber MR, Fell R, Mason W. Use of wrist activity for monitoring sleep/wake in demented nursing-home patients. Sleep. 1997;20(1):24-27.

24. Brooks JO, Friedman L, Bliwise DL, Yesavage JA. Use of the wrist actigraph to study insomnia in older adults. Sleep. 1993;16(2): 151-155.

25. Chu LW, Chiu KC, Hui SL, Yu GK, Tsui WJ, Lee PW. The reliability and validity of the Alzheimer's Disease Assessment Scale Cognitive Subscale (ADAS-Cog) among the elderly Chinese in Hong Kong. Ann Acad Med Singapore. 2000;29(4):474-485.

26. Mohs RC. The Alzheimer's Disease Assessment Scale. Int Psychogeriatr. 1996;8(2):195-203.

27. Diagnostic and Statistical Manual of Mental Disorders, Fourth Edition, Text Revision (DSM-IV-TR). Washington, DC: American Psychiatric Association; 2000.

28. Chen ML, Lin LC, Wu SC, Lin JG. The effectiveness of acupressure in improving the quality of sleep of institutionalized residents. J Gerontol A Biol Sci Med Sci. 1999;54(8):M389-M394.

29. Hopton A, Thomas K, Macpherson H. The acceptability of acupuncture for low back pain: a qualitative study of patient's experiences nested within a randomised controlled trial. PLoS One. 2013;8(2):e56806.

30. [No authors listed]. NIH Consensus Conference. Acupuncture. JAMA. 1998;280(17):1518-1524.
Clinical Interventions in Aging

\section{Publish your work in this journal}

Clinical Interventions in Aging is an international, peer-reviewed journal focusing on evidence-based reports on the value or lack thereof of treatments intended to prevent or delay the onset of maladaptive correlates of aging in human beings. This journal is indexed on PubMed Central, MedLine, the American Chemical Society's 'Chemical Abstracts

\section{Dovepress}

Service' (CAS), Scopus and the Elsevier Bibliographic databases. The manuscript management system is completely online and includes a very quick and fair peer-review system, which is all easy to use. Visit http://www.dovepress.com/testimonials.php to read real quotes from published authors. 